\title{
Fabrication of quantum dots, metal nanostructures and 2D nanocomposites materials for enhanced photo-catalytic and nonlinear optical properties for applications in future photonic devices
}

\author{
P. Kumbhakar*, S. Biswas and A. K. Kole \\ Nanoscience Laboratory, Dept. of Physics, \\ National Institute of Technology, Durgapur, 713209, West Bengal, India \\ *Email: pathik.kumbhakar@phy.nitdgp.ac.in, nitdgpkumbhakar@yahoo.com
}

Low dimension materials have drawn huge attention of researchers due to their unique properties arising due to the quantum confinement effect. $\mathrm{Mn}^{2+}$ or $\mathrm{Cu}^{2+}$ doped $0 \mathrm{D} \mathrm{ZnS}$ quantum dots (d-dots) [1] or 1D silver nanowire (AgNWs) and 2D hexagonal boron nitride (hBN) [2] and graphene oxide (GO), $\mathrm{ZnS}$ nanosheet and its composites have been studied extensively for their tunable photoluminescence (PL) and enhanced nonlinear optical (NLO) properties [15]. Tunable dual emissions in UV and yelloworange regions have been reported by us, just by varying $\mathrm{Mn}^{2+}$ concentration in $\mathrm{ZnS}$ d-dots. Also tunable visible PL emissions have been obtained by varying the $\mathrm{Cu}^{2+}$ doping concentration $(0-1 \%)$ in $\mathrm{ZnS}$ d-dots. A charge transfer mechanism from host to dopant has been provided to explain the observed tunability in PL emission in ZnS:Mn ddots. Figure 1 (upper panel) shows a typical PL emission spectra obtained from synthesized undoped $\mathrm{ZnS}, \mathrm{Cu}^{2+}$ doped and $\mathrm{Mn}^{2+}$ doped $\mathrm{ZnS}$ d-dots.

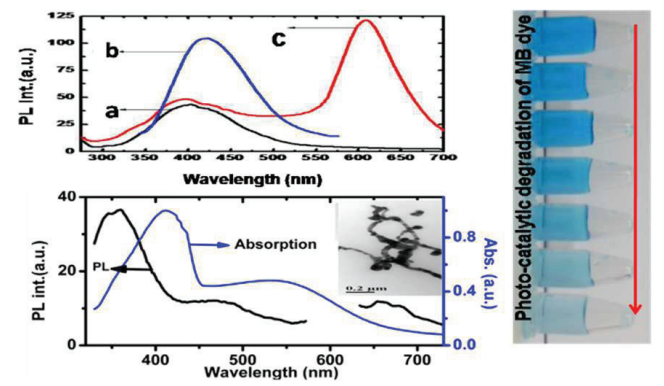

Figure 1: Upper panel shows the PL spectra of (a) ZnS, (b) $\mathrm{ZnS}: \mathrm{Cu}(1.00 \%)$ and (c) ZnS:Mn (40.0\%). The lower panel is the absorption and PL emission (ex$330 \mathrm{~nm})$ spectra of the AgNWs. Photo-catalytic degradation of Methylene Blue (MB) dye by $\mathrm{ZnO}-\mathrm{ZnS}$ nanocomposite is shown in the right column

Also we have synthesized multi-plasmonic shapes [3] of silver nanoparticles by varying reaction temperature only in a simple chemical method. At $90^{\circ} \mathrm{C}$ temperature, we have obtained 1D AgNWs of several $\mu \mathrm{m}$ in length, which shows two absorption bands corresponding to surface Plasmon resonance (SPR) and also the sample shows distinct PL emission due to the splitting of primary SPR enhanced PL emission (Figure 1, lower panel).

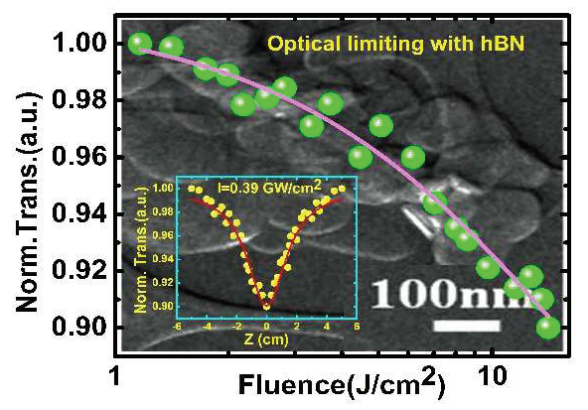

Figure 2: OL effect of hBN and Inset shows a typical OA Z-scan traces (background - TEM image)

Wurtzite (WZ) ZnS nanosheets, $\mathrm{ZnS}-\mathrm{ZnO}$ composite, and fungi-like $\mathrm{ZnO}$ nanostructures have also been synthesized and studied their photo-catalytic performances. It has been found that $\mathrm{ZnO}-\mathrm{ZnS}$ composites show highest degradation efficiency of $74 \%$ due to the formation of surface complex and higher visible light absorption (Figure $12^{\text {nd }}$ column). The NLO properties of $\mathrm{hBN}$ and GO have also been investigated by the open and closed aperture Zscan techniques and both the materials show optical limiting (OL) effects (Figure 2) and may find potential applications in laser related military safety devices.

\section{References}

1. A. K. Kole, C. S. Tiwary, P. Kumbhakar, J. Appl. Phys. 113 (2013) 114308

2. A. K Kole, P. Kumbhakar, U. Chatterjee. Appl. Phys. Lett. 100 (2012) 013103.

3. S. Biswas, A. K. Kole, R. Sarkar, P. Kumbhakar. Mater. Res. Exp. 1 (2014) 04504.

4. A. K. Kole, C. S. Tiwary, P. Kumbhakar. J. Mater. Chem. C. 2 (2014) 4338.

5. P. Kumbhakar et al. Adv. Opt. Mater. 3 (2015) 828. 